\title{
CARCINOMA BILATERAL DO PLEXO CORÓIDE OPERADO COM SUCESSO
}

\author{
Gilberto M. DE Almeida * \\ Charles P. Tilbery** \\ Luiz G. Gagliardi-Ferreira **
}

Os tumores do plexo coróide (TPC) são usualmente classificados em carcinomas e papilomas, de acôrdo com o seu aspecto histológico; entretanto, sabe-se que o prognóstico relaciona-se mais com o aspecto infiltrativo do que com as características de malignidade celular ${ }^{2,16,8}$. Para Monteiro e Lopes ${ }^{8}$ os carcinomas devem ser considerados como carcinomas papiliferos pré-invasivos.

A incidência de TPC é de aproximadamente $0,5 \%$ entre todos os tumores intracranianos 4, 7, 10 mas, em crianças, atinge a 3,9\% 7 . Em adultos os TPC são mais freqüentes no quarto ventriculo; em crianças são mais encontrados em um dos ventrículos laterais, raramente em ambos.

Ray e Pech (1956) ' relataram o caso de criança com hidrocéfalo por aumento da produção do líquido cefalorraqueano (LCR), causado por papiloma bilateral nos ventrículos laterais; após três tentativas frustadas de drenagem do LCR (para o ureter e peritônio) foram feitas duas craniotomias; a criança faleceu em choque, durante o transcurso da segunda craniotomia, após retirada do tumor; na necrópsia não foram encontrados restos tumorais. Matson e Crofton (1960) ${ }^{7}$, em ampla revisão sôbre TPC, encontraram 6 casos bilaterais; na casuística dêstes autores, entretanto, não há referência a tumores bilaterais. Baar e Galindo (1964) ${ }^{3}$ apresentaram um caso no qual os papilomas dos dois ventrículos laterais foram encontrados mediante necropsia. Entre os 6 doentes de Teng e Papatheodorou (1966) ${ }^{11}$, um faleceu 6 meses após a intervenção cirúrgica devido à existência de tumor semelhante no lado oposto.

Justifica-se a publicação dêste trabalho pelo fato de não têrmos encontrado, na literatura que estêve a nosso alcance, caso de carcinoma bilateral do plexo coróide operado com sucesso.

* Neurocirurgião, Departamento de Neurologia da Faculdade de Medicina da Universidade de São Paulo; ** Médicos estagiários, Secção de Neurologia, Faculdade - de Ciências Médicas da Santa Casa de São Paulo. 
O B S E R V A C A O

N. P. S., 11 anos, sexo masculino, branco, brasileiro, examinado no dia 1 de novembro de 1969. O paciente queixava-se de cefaléias periódicas há dois anos. Nas últimas semanas tornou-se confuso e a dôr de cabeça intensificou-se. O exame mostrou sonolência e papiledema bilateral. Radiografias do crânio: disjunção de suturas. Eletrencefalograma (EEG) normal. Angiografia pela artéria carótida direita (fig. 1): visualização de ambas as artérias cerebrais posteriores; sinais de dilatação ventricular, principalmente do corno temporal; artéria cerebral anterior desviada para a esquerda e vasos silvianos estirados e elevados; na região atrial, área de circulação patológica irrigada pelas artérias coroidéias anterior e posterior, mais evidente na fase venosa; suspeita da existência de outro tumor no lado esquerdo, em algumas chapas em incidência antero-posterior. Angiografia cerebral pela artéria vertebral direita (fig. 2): ambas as artérias coroidéias posteriores suprem os respec-
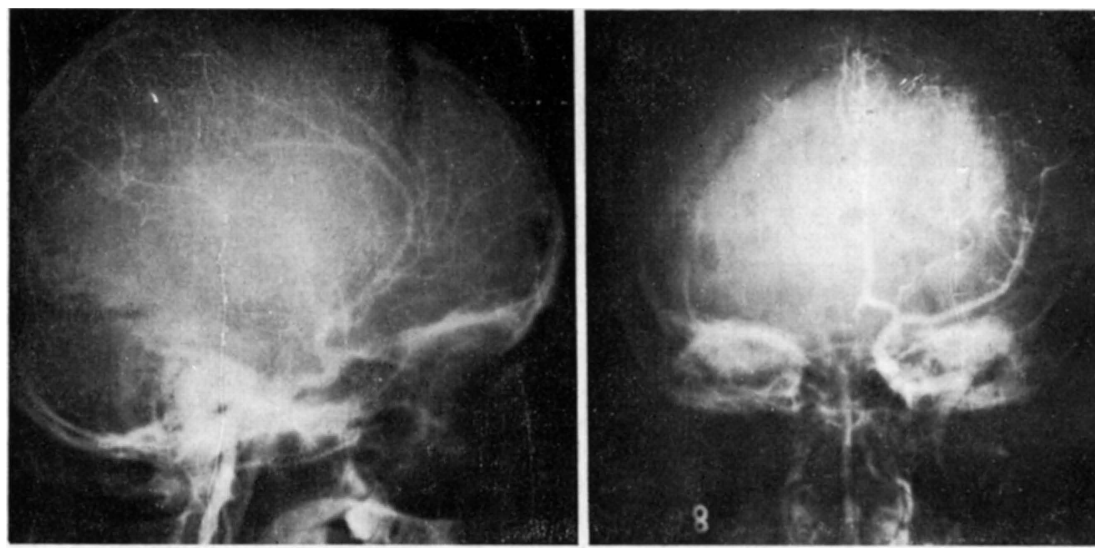

Fig. 1 - Caso N.P.S. Arteriografia carotídea direita mostrando discreto desvio da artéria cerebral anterior, vizibilização de ambas artérias cerebrais posteriores e área de circulação patológica suprida pelas artérias coroidéias anterior e posterior.

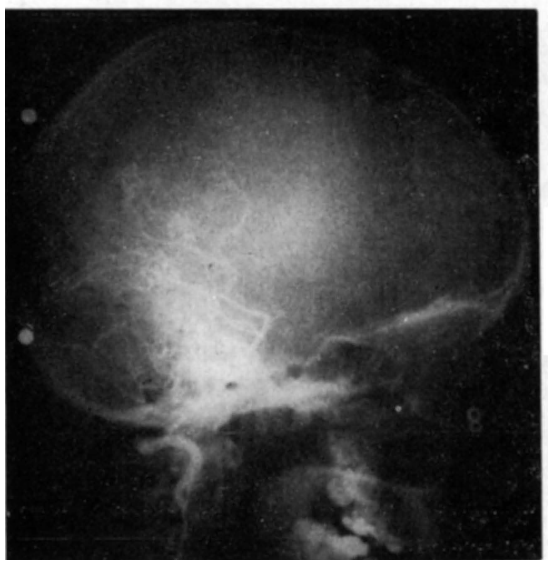

Fig. 2-Caso N.P. S. Arteriografia pela vertebral mostrando ambas artérias coroidéias posteriores irrigando os tumores. 
tivos tumores. Angiografia cerebral via carótida esquerda (fig. 3): melhor visibilizaçāo do tumor do lado esquerdo, a artéria coroidéia anterior contribui na sua circulação.
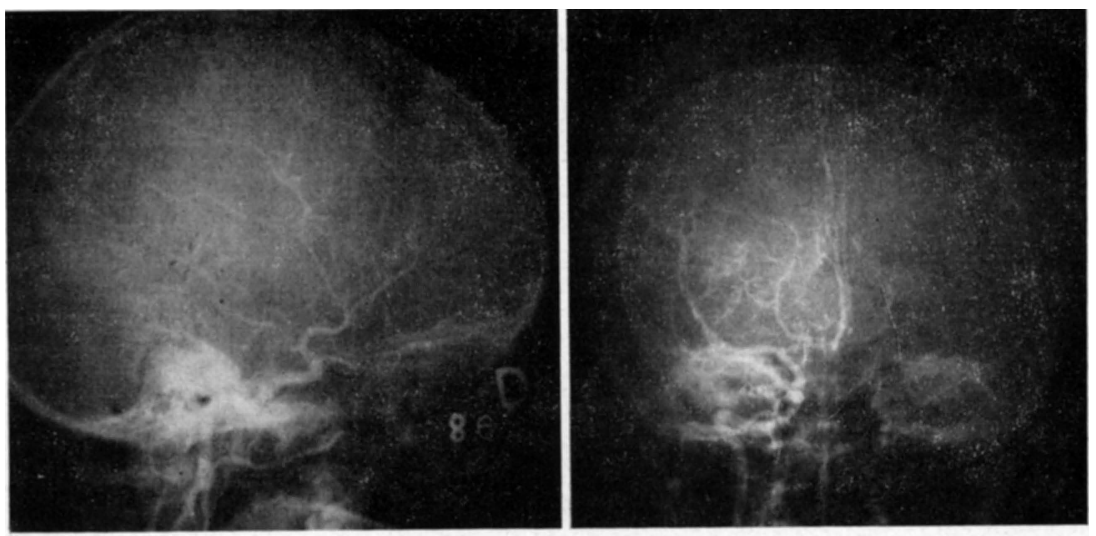

Fig. 3 - Caso N.P.S. Arteriografia carotidea esquerda mostrando a área de circulação patológica.

Intervenção cirúrgica - Ampla craniotomia parieto-occipital direita em 9-11-1969. Mediante incisão cortical foi exposto grande tumor no ventriculo lateral. $\mathrm{Na}$ impossibilidade de mobilizar a neoplasia para clipar o pedículo, o tumor foi retirado por aspiração. No pós-operatório a criança ficou torporosa, mas sem sinal de localização. Após curto periodo de melhora o paciente voltou a piorar, provàvelmente pelo desiquilibrio provocado pela presença de tumor no lado oposto. A neoplasia do ventriculo esquerdo foi aspirada em uma segunda craniotomia, realizada em 3 de dezembro. Após o segundo ato cirúrgico o doente recuperou a consciência progressivamente. No fim de dois meses apresentava-se apenas com déficit parcial de visão, que persiste até hoje (um ano de seguimento pós-operatório).

O estudo histopatológico de ambos os tumores confirmou a hipótese de TPC, sendo o diagnóstico de carcinoma firmado devido ao poimorfismo celular, às células multinucleadas e às mitoses encontradas.

\section{O M E N T $\AA$ R I O S}

Os sintomas de TPC variam de caso para caso; no nosso doente eram devidos apenas à hipertensão intracraniana. As radiografias simples do crânio e o EEG não contribuiram para o diagnóstico.

A angiografia pela carótida direita mostrou aspecto típico de TPC, de acôrdo com Almeida e Barros ${ }^{2}$ : presença de circulação patológica suprida pelas artérias coroidéias, discreto desvio da artéria cerebral anterior e elevação dos vasos do gupo silviano (sugerindo bloqueio do corno temporal). Casualmente, 0 contraste alcançou as artérias cerebral posterior e coroidéia posterior do lado oposto, permitindo a visibilização, em incidência antero-posterior, do tumor do lado esquerdo. O estudo complementar, pela angio- 
grafias vertebral e carotídea esquerda, confirmou a presença de uma neoplasia em cada lado, supridas pelas artérias coroidéias anteriores e posteriores. Há a possibilidade de não se fazer o diagnóstico, em outros casos de tumores bilaterais, se a angiografia fôr realizada apenas de um lado. Concluímos, pois, que é necessário o estudo angiográfico bilateral, quando há suspeita de TPC.

A pneumoventriculografia é recomendada por alguns autores ${ }^{5,10}$ para diagnóstico de TPC. Em trabalho anterior ${ }^{2}$ um de nós já defendeu a opinião de que o estudo angiográfico é mais aconselhável. Em tumores bilaterais as angiografias contribuem melhor para completa elucidação diagnóstica, como foi demonstrado no presente caso.

A mortalidade cirúrgica, mesmo nos casos com tumor unilateral, é elevada. Matson e Crofton ${ }^{7}$ relataram uma mortalidade de $28,5 \%$ em sua experiência mas, a revisão de literatura forneceu um indice de $50 \%$. Bohm e Strang ${ }^{4}$ apresentaram 8 casos, dos quais 5 faleceram $(62,5 \%)$. Aicardi e col. ${ }^{1}$, em revisão, encontraram 22 óbitos num grupo de 57 pacientes operados $(38,6 \%)$, e mais 4 casos fatais devido à recorrência $(7,0 \%)$.

No que se refere à técnica cirúrgica, Matson e Crofton ${ }^{7}$ afirmam: "Na remoção dêstes tumores é feita uma tentativa de mobilizar a neoplasia e interromper imediatamente seu suprimento sangüineo através do plexo coróide. Desde que isto seja conseguido, o tumor diminui muito de volume e usualmente pode ser removido intacto. $\dot{E}$ importante não abordar a neoplasia antes de individualizar seu pedículo; caso contrário a hemorragia torna-se de difícil contrôle". Para Lawrence, Hoare e Till ${ }^{5}$ o melhor método seria o de remover o tumor intacto, mas reconhecem a dificuldade de clipar os vasos do pedículo. Nós acreditamos que, em tumores grandes, a técnica utilizando aspiração é menos traumática.

\section{R E S U M O}

Os autores não encontraram na literatura caso de carcinoma bilateral do plexo coróide operado com sucesso. O trabalho consta da apresentação de uma criança de 11 anos com carcinoma dos plexos coróides nos dois ventrículos laterais. São feitas considerações a respeito das dificuldades no diagnóstico, da importância do estudo angiográfico completo e do tratamento cirúrgico.

S U M M A R Y

\section{Bilateral carcinoma of the choroid plexus operated successfully}

A case of bilateral carcinoma of the choroid plexus is reported. Bilateral carcinoma of the choroid plexus are very rare and the authors did not find in the literature a similar case operated sucessfully. The difficulties for dignosis are discussed. The possibility exists that in other cases with bilateral tumors a complete diagnosis is impossible if angiography is performed 
only in one side. Therefore, it is necessary to perform a bilateral angiographic study when a tumor of the choroid plexus is suspected. The surgical treatment and the mortality rate are also discussed.

\section{REFER N C I A S}

1. AICARDI, J.; GARCIA, J.; LEPINTRE, J.; CHEVRIE, J. J. \& THIEFFRY, St. - Les papillomes des plexus choroides chez l'enfant. Arch. franc. Pédiat. $25: 673,1968$.

2. ALMEIDA, G. M. \& BARROS, N. G. - Diagnóstico angiográfico dos tumores do plexo coróide do ventrículo lateral. Arq. Neuro-Psiquiat. (São Paulo) 24: $103,1966$.

3. BAAR, E. \& GALINDO, J. - Bilateral papilloma of the choroid plexus. J. Maine med. Ass. 55:95, 1964.

4. BOHM, E. \& STRANG, R. - Choroid plexus papillomas. J. Neurosurg. 18: 493, 1961.

5. LAURENCE, K. M.; HOARE, R. D. \& TILL, K. - The diagnosis of the choroid plexus papilloma of the lateral ventricle. Brain 84:628, 1961.

6. LEWIS, P. - Carcinoma of the choroid plexus. Brain 90:177, 1967.

7. MATSON, D. D. \& CROFTON, F. D. - Papilloma of the choroid plexus in childhood. J. Neurosurg. 17:1002, 1960.

8. MONTEIRO, D. M. \& LOPES, Z. A. - Neoplasias papilíferas do plexo coróide na infância. Revisão da literatura e apresentação de 4 casos. Rev. Hosp. Clín. Fac. Med. São Paulo 23:78, 1968.

9. RAY, B. \& PECK, F. C. - Papilloma of the choroid plexus of the lateral ventricles causing hydrocephalus in an infant. J. Neurosurg. 13:405, 1956.

10. TAVERAS, J. M. \& WOOD, E. H. - Diagnostic Neuroradiology. Williams \& Wilkins Co., Baltimore, 1964.

11. TENG, P. \& PAPATHEODOROU, C. - Tumors of the cerebral ventricles in children. J. nerv. ment. Dis. 142:87, 1966.

Rua Itapeva 490, 9.0 andar, conj. 96 - São Paulo, ZP 3 - São Paulo - Brasil. 